\title{
BERICHT ZUR 2. INFORMATIONSVERANSTALTUNG DES OPEN ACCESS NETWORK AUSTRIA (OANA) (WIEN, 21. JÄNNER 2015)
}

\section{von Katrin Buschmann und Katharina Rieck}

Zusammenfassung: Dieser Beitrag fasst die Ergebnisse der 2. OANA-Veranstaltung am 21.01.2015 im Palais Harrach in Wien zusammen. Das „Open Access Network Austria" (OANA) ist eine ,joint activity" unter dem organisatorischen Dach des Fonds zur Förderung der wissenschaftlichen Forschung (FWF) und der Österreichischen Universitätenkonferenz (UNIKO) mit dem Ziel Open Access Aktivitäten in der österreichischen Forschungslandschaft zu koordinieren. Nach einer ersten OANA-Informationsveranstaltung im November 2013 wurden fünf Arbeitsgruppen zu folgenden Themen gebildet: Open Access Policy, Finanzierung von Open Access, Rechtliche und politische Rahmenbedingungen, Publikationsmodelle und Einbindung von WissenschafterInnen. Im Rahmen der zweiten Informationsveranstaltung wurden die Ergebnisse bzw. Fortschritte der fünf Arbeitsgruppen präsentiert. Mit der Weiterführung von OANA sollen zukünftig einige der genannten Bereiche vertieft sowie um neue Themenfelder ergänzt werden.

Schlüsselwörter: Open Access, Open Access Network Austria (OANA), Fonds zur Förderung der wissenschaftlichen Forschung (FWF), Universitätenkonferenz (UNIKO), wissenschaftliche Publikationsmodelle, e-infrastructures Austria

\section{REPORT OF THE $2^{\text {nd }}$ OPEN ACCESS NETWORK AUSTRIA (OANA) INFORMATION EVENT (VIENNA, 21 ${ }^{\text {st }}$ JANUARY 2015)}

Abstract: This article provides an overview of the activities at the second OANA meeting at the Palais Harrach in Vienna on the 21st of January, 2015. OANA is a joint activity initiated and organized by the Austrian Science Fund (FWF) and the Universities Austria (UNIKO) that aims to coordinate Open Access activities in the Austrian research landscape. After a kick-off meeting in November 2013, five expert groups worked on the following topics: Open Access Policy, Financing Open Access, the Legal and Political Framework, Publication Models and Engagement of Researchers. Their findings were presented at the second OANA information meeting. In the future, OANA will continue working on some of these topics and will broaden its fields of interest.

Keywords: Open Access, Open Access Network Austria (OANA), Austrian Science Fund (FWF), Universities Austria (UNIKO), scholarly communication, e-infrastructures Austria 
Das „Open Access Network Austria“ ( $\underline{\text { OANA) }}$ ist eine ,joint activity“ unter dem organisatorischen Dach des Fonds zur Förderung der wissenschaftlichen Forschung (FWF) und der Österreichischen Universitätenkonferenz (UNIKO). Es vereinigt 49 Institutionen mit über 60 Ansprechpersonen unter seinem Dach.

Das Netzwerk, das vom FWF koordiniert wird, knüpft an die Open-AccessEmpfehlungen der UNIKO vom Januar 2010 und ein Positionspapier des FWF von 2013 an und hat sich folgende Ziele gesetzt:

- Abstimmung der Empfehlungen zu Open-Access-Aktivitäten der österreichischen Forschungsstätten, Fördergeber und Forschungspolitik (inkl. der Berücksichtigung internationaler Entwicklungen)

- Positionierung gegenüber den Informationsanbietern (v.a. Verlage)

- Ansprechpartner und Informationsquelle für Wissenschafterlnnen, Forschungsstätten und (Forschungs-)Politik

Anfang 2014 wurden fünf aus Open-Access-ExpertInnen bestehende Arbeitsgruppen eingerichtet. Ziel war die Ausarbeitung konkreter Empfehlungen an die OANA-Mitglieder zur Entwicklung einer effizienten Open-Access-Strategie für die Akteure des Wissenschaftssystems. Fortschritte und Ergebnisse der Arbeitsgruppen wurden am 21.01.2015 im Rahmen der 2. OANA-Informationsveranstaltung mit 77 Teilnehmerlnnen präsentiert und diskutiert.

Bereits während der Einführungsvorträge von Falk Reckling (FWF) „Internationale und nationale Entwicklungen" und Peter Seitz (BMWFW) „Open Access Network Austria - Die Sicht des BMWFW“ wurde deutlich, dass das Thema Open Access sowohl national als auch international weiter sehr stark an Bedeutung gewinnt.

Die vom FWF durchgeführte Scientists Survey 2013 bestätigt dies. $67,9 \%$ der befragten Wissenschafterlnnen beurteilen das Thema „Open Access“ als „wichtig“ bis „sehr wichtig“. Vor allem junge Wissenschafterlnnen (79,5\%) sehen einen hohen bis sehr hohen Bedarf, Open Access in Österreich zu fördern.

Rasante Entwicklungen in vielen EU-Mitgliedsstaaten wie Schweden, Deutschland, Dänemark, Großbritannien oder den Niederlanden sowie eine Empfehlung der Europäischen Kommission lassen einen internationalen Durchbruch für Open Access erwarten. Diese Ambition hat auch jüngst wieder der EU-Kommissar für Forschung, Wissenschaft und Innovation Carlos Moedas unterstrichen.

Braucht Österreich demnach ein größeres politisches (inkl. finanzielles) Commitment im Rahmen einer nationalen Strategie und mit überprüfbaren Benchmarks? 
Wie kann das Ziel der RFTE-Strategie 2020, bis zum Jahr 2020 alle öffentlichen Forschungsergebnisse in Österreich frei im Internet zugänglich zu machen, umgesetzt werden?

Das BMWFW sieht eine Reihe von Aktivitäten und belastbaren Netzwerken, die historisch und „bottom-up“ gewachsen sind. Gerade deshalb funktionieren sie über Ressortgrenzen hinweg, autonom und zum Teil ohne gesetzliche Grundlage. Aus den einzelnen Policies der Netzwerke entwickelt sich eine Politik für Österreich.

Auf nationaler Ebene möchte das Ministerium den hohen Autonomiegrad der Institutionen respektieren und ist in der Entwicklung dieser Politik auf deren autonome ExpertenInnen angewiesen. Open Access soll im Rahmen der Leistungsvereinbarung zum Thema gemacht werden.

Auf EU-Ebene möchte das Ministerium den Beitrag Österreichs in den NPR Meetings und der ERA Working Group einbringen und das OANANetzwerk zur Weitergabe von Informationen nutzen.

Die Summe der verschiedenen Aktivitäten könnte die Geburtsstunde einer gemeinsamen Strategie sein. Durch bessere Bündelung bereits gebundener (finanzieller) Ressourcen und einer koordinierten Vorgehensweise könnte Österreich auf europäischer Ebene zum „innovation leader“ werden. Dabei hätte Österreich als kleines Land entscheidende Vorteile.

Im Folgenden wurden die Ergebnisse der Arbeitsgruppen präsentiert und diskutiert.

\section{Arbeitsgruppe: Open Access Policy}

Bruno Bauer (UB Med Uni Wien), Andreas Ferus (UB Akademie der bildenden Künste Wien) und Lisa Schilhan (UB Uni Graz) präsentierten die Ergebnisse der Arbeitsgruppe „Open Access Policy“. Ziel war die Entwicklung eines modularen Templates für eine Open Access Policy, das auf die z.T. sehr unterschiedlichen Bedürfnisse und Orientierungen der österreichischen Forschungsinstitutionen Rücksicht nimmt. Als Ergebnis wurden zwei Checklisten vorgestellt:

1. Checkliste zur Entwicklung einer institutionellen Open Access Policy

2. Checkliste zu institutionellen Förderungsmaßnahmen von Open Access nach der Implementierung einer Open Access Policy

Bei der Entwicklung einer OA-Policy sollten u.a. folgende Standards eingehalten werden:

- Als HTML- und als PDF-Dokument auf der Website verfügbar

- sowohl auf Deutsch als auch auf Englisch 
Die Benefits einer OA-Policy für die Wissenschafterlnnen müssen klar kommuniziert werden, der Grad der Verbindlichkeit der Policy muss überprüft werden. Es ist nötig, diese jährlich zu evaluieren bzw. weiter zu entwickeln. Forschungsdaten bedürfen einer eigenständigen Policy.

Institutionelle Maßnahmen nach der Entwicklung einer OA-Policy hängen stark von der gewählten OA-Strategie ab. Fragen in den Bereichen Personalmanagement, Organisation, Infrastruktur, Finanzierung etc. sind vor dem obligatorischen Einsatz eines OA-Beauftragten zu klären. Am Beispiel einer ausgearbeiteten Stellenbeschreibung wurden nötige Qualifikationen eines OA-Beauftragten beschrieben.

Betont wurde, dass den Wissenschafterlnnen das OA-Publizieren durch die jeweilige Institution ermöglicht werden muss. Der Grüne Weg sieht hierfür den Aufbau eines institutionellen Repositoriums vor. Wird der Goldene Weg angestrebt, ist eine Plattform für eigene Gold-OA-Zeitschriften anzubieten und ein Publikationsfonds für Gold-OA-Artikel einzurichten.

Erfahrungen mit dem Grünen und Goldenen Weg wurden anhand des Best-Practice-Beispiels UB Uni Graz dargestellt. Einige wichtige Eckpunkte wurden herausgegriffen:

- Gruppenarbeit bzw. Dialog zwischen allen relevanten Akteurlnnen der Institution

- Schaffung des zeitlichen Raumes

- Zur-Verfügung-Stellung finanzieller Mittel für Weiterbildung

- Rechtsberatung von Wissenschafterinnen nur durch Juristln möglich

Die Frage nach der Notwendigkeit einer bibliothekarischen Ausbildung der OA-Beauftragten wird als wesentlich betrachtet, da sich Bibliotheken von jeher nicht nur für die Sammlung, sondern auch die Verteilung von Informationen verantwortlich zeichnen.

\section{Arbeitsgruppe: Einbeziehung von WissenschafterInnen}

Die Arbeitsgruppe „Einbeziehung der Wissenschafterlnnen“ - repräsentiert durch Michael Nentwich (ÖAW - ITA), Peter Kraker (KNOW Center Graz), Veronika Gründhammer (Uni Innsbruck), Michela Vignoli ( $\underline{A I T})$ und Michael Strassnig (WWTF) - erarbeitete fünf Open-Access-Konzepte aus Nutzerlnnen-Perspektive:

1. INFO: Allgemeines Informationsblatt

2. SUPPORT: Hilfestellung bei der Umsetzung

3. ÖA: Involvierung der Öffentlichkeitsarbeit 


\section{VISIBILITY: Label Bekenntnis zu OA \\ 5. TECHNIK: Softwareentwicklung}

Ein allgemeines Informationsblatt soll zielgruppen- und institutionsspezifisch den Nutzen von Open Access aus Sicht der Wissenschafterlnnen veranschaulichen. Auch diese Arbeitsgruppe empfiehlt eine spezifische OA-Ansprechperson. Contact Points zur individuellen Beratung und für Schulungen von Wissenschafterlnnen sollen geschaffen werden. Dabei könnte OANA sowohl bei der Erarbeitung des allgemeinen Informationsblattes als auch bei der Erstellung von Schulungsmaterialien unterstützen und Unterlagen zur Verfügung stellen. Eine Integration von Informationen zu Open Access in die Studienpläne (z.B. „Einführung in das wissenschaftliche Arbeiten") soll erfolgen. Die jeweiligen Abteilungen für Öffentlichkeitsarbeit müssen direkt eingebunden werden, um eine professionelle textuelle und grafische Aufarbeitung sowie eine effektive institutionelle Öffentlichkeitsarbeit zu gewährleisten. Open Access soll mittels eines Labels besser sichtbar gemacht werden. Die Wissenschafterlnnen sollen sich im Rahmen einer Kampagne (z.B. „Ich bin Open Access“) offen zu Open Access bekennen können. Allerdings wurde von den Diskutantlnnen im Anschluss angemerkt, dass ein solches Bekenntnis international - also nicht nur deutschsprachig - sein sollte, und dass bereits eine solche Plattform (iopenaccess.com) existiert.

Abschließend wurden fünf Software-Ideen präsentiert:

1. Ubiquitäres OA-Label (Symbol zu jeder OA-Publikation samt hinterlegtem Download-Link)

2. Qualitätsstandards für OA-Repositorien

3. Rückmeldungen zur Nutzung von OA Publikationen (z.B. Altmetrics)

4. Metasuche zur OA Verfügbarkeit (OAI-Schnittstellen z.B. zu Mendeley, ResearchGate, academia.edu etc. zur OA-Verfügbarkeitsüberprüfung)

5. Vernetzung mit geschlossenen Repositorien (über APIs Metadaten extrahieren, um sie in die Metasuche zu integrieren)

Vor allem zum Thema Metasuche ist die Frage nach rechtlicher Deckung noch nicht ansatzweise geklärt. Es wird kritisiert, dass die genannten „Sozialen Netzwerke“ (Mendeley u.a.) durch eine obligatorische Registrierung nicht den OA-Kriterien entsprechen und dass Elsevier bereits 1.800 Personen mittels Unterlassungsklage zur Entfernung aller Publikationen aus solchen Netzwerken zwingen konnte. 


\section{Arbeitsgruppe: Rechtliche und politische Rahmenbedingungen}

Peter Seitz (BMWFW), Ulrike Kortschak (UB Med Uni Graz) und Anton Graschopf (RFTE) präsentierten im Anschluss die drei Themenkomplexe, die im Zentrum der Arbeitsgruppe „Rechtliche und politische Rahmenbedingungen" standen: Monitoring von Open Access an Universitäten, das Urheber-/Zweitveröffentlichungsrecht und die österreichische Publikationsinfrastruktur. Durch eine vom Ministerium im Juli 2014 durchgeführte Umfrage zum Thema Awareness von Open Access an österreichischen Universitäten konnte aufgezeigt werden, dass Open Access ein sehr wichtiges Thema für die Forschungsstätten ist. Besonders die Repositorienlandschaft in Österreich, an der auch fünf europäische Universitäten teilnehmen, das Projekt e-infrastructures Austria sowie die KEMÖ, wurden vom Vertreter des Ministeriums ausdrücklich als erfolgreiche österreichische Initiativen bezeichnet. Weiters wurde das Zweitveröffentlichungsrecht als besonders wichtig in der Diskussion um OA hervorgehoben. Es sollte für jede wissenschaftliche Publikation, die mit öffentlichen Mitteln gefördert bzw. finanziert wird gelten und zum einfachen Nutzungsrecht der UrheberInnen zur Zweitveröffentlichung im Internet führen. Dazu müsste der AG zufolge allerdings die Umsetzung einer forschungspolitischen Strategie in diesem Bereich angestrebt bzw. gesetzliche Rahmenbedingungen geschaffen werden. Auch unterschiedliche OA-Publikationsmodelle an Universitäten - z.B. eine "gemeinsame Publikationsplattform" oder eine gemeinsame OA-Publikationsfinanzierung - sollten durch die Forschungspolitik Förderung erfahren.

\section{Arbeitsgruppe: Finanzierung von Open Access}

Die AG „Finanzierung von Open Access“ - vertreten durch Falk Reckling ( $\underline{F W F})$ und Brigitte Kromp (KEMÖ) - präsentierte anschließend erste Schätzungen zum Publikationskostenvolumen in Österreich sowie eine Übersicht über die Verhandlungen mit den Verlagen.

\section{Publikationskosten}

Für Subskriptionsmedien (Zeitschriften und z.T. auch Datenbanken) beliefen sich Anschaffungskosten für die Universitäten inkl. einiger Fachhochschulen für das Jahr 2013 auf rund EUR 30 Millionen. Zusätzlich zu den Subskriptionspreisen kommen dazu noch etwa EUR 1,5 Mio. an Autorlnnengebühren (u.a. page charges, submission fees, colour figures). 
Im Bereich Open Access fallen an AutorInnengebühren für Hybrid Open Access ca. EUR 2,4 Mio. (davon 90\% FWF) und für Gold Open Access ca. EUR 1,5 Mio. (davon ca. 30\% FWF) an.

Schließlich gibt es noch eine Reihe an direkten und indirekten Zuschüssen für wissenschaftliche Publikationen, die sich bisher nicht oder nur mit großem Aufwand erheben lassen.

\section{Verlagsverhandlungen}

Ein zentrales Strategieelement sind in Österreich Verhandlungen mit Verlagen, die einen Übergang zu Open Access ermöglichen. Hier hat Österreich neben den Niederlanden und Großbritannien eine Vorreiterrolle. Dazu zählen etwa Vereinbarungen mit der Royal Society of Chemistry, die Beteiligung an $\underline{\mathrm{SCOAP}^{3}}$ oder die Abschlüsse mit loP Publishing und mit Taylor \& Francis.

Die Arbeitsgruppe kam zu zwei Empfehlungen:

1. Vertiefung der Arbeitsgruppe zum Thema Erhebung, Koordination und Administration von Publikationskosten in Österreich inkl. potentieller Einsparungsmöglichkeiten.

2. Weiterführung der Verhandlungen mit Verlagen wie Wiley und Springer, die einen vollständigen Umstieg auf Open Access ermöglichen (siehe das Beispiel Niederlande).

Das Thema alternative Finanzierungsmodelle (Sylvia Petrovic-Majer, OFKN) wurde aufgrund des Umfangs nicht präsentiert, wird aber in der verschriftlichten Fassung vorliegen.

\section{Arbeitsgruppe: Open Access Publikationsmodelle}

Die Arbeitsgruppe „Open Access Publikationsmodelle“ erarbeitete, die bereits auf OANA.at publizierte "Checkliste für die Herausgabe von Open-Access-Zeitschriften an Forschungseinrichtungen", die von Guido Blechl (UB Wien) vorgestellt wurde. Sie basiert u.a. auf den Erfahrungen, die bereits Forschungsstätten wie die Universitäten Wien und Graz sowie der FWF bei der Herausgabe bzw. Förderung von Open Access Zeitschriften gemacht haben. Die Checkliste soll interessierte Wissenschafterlnnen und österreichische Forschungseinrichtungen Schritt für Schritt durch den Prozess der Implementierung einer OA-Zeitschrift führen. 


\section{e-infrastructures Austria - Aktueller Stand}

Im Anschluss wurde das im Jänner 2014 gestartete, dreijährige, vom BMWFW finanzierte Partnerprojekt e-Infrastructures Austria von Eva Ramminger (UB TU Wien) und Patrick Danowski (IST Austria) präsentiert. Mitarbeiterlnnen aus 25 wissenschaftlichen Einrichtungen arbeiten dabei im Rahmen von 12 Arbeitsclustern zusammen.

Das Projekt wurde initiiert, um der steigenden Relevanz von OA in der Wissenschaftscommunity Rechnung zu tragen. Unsicherheiten im Umgang mit Forschungsdaten sollen damit ausgeräumt werden, und eine klare Positionierung von UNIKO und BMWFW im Hinblick auf OA und Forschungsdaten soll erreicht werden. Ein Finanzierungs- und Kooperationsmodell durch den Hochschulraumstrukturmittel-Fonds soll etabliert werden.

Die Ziele des Projekts umfassen den Aufbau von Repositorien zur Sichtbarmachung von Forschungsergebnissen, die Erarbeitung von Standards und Workflows zur Sammlung und Integration von Forschungs(roh)daten und die Schaffung von Wissensnetzwerken. Dabei profitieren WissenschafterInnen, Förderer, Bibliotheken und IT-Services der ProjektpartnerInnen gleichermaßen.

Mit diesem zentralen Infrastrukturprojekt für die österreichische Forschungslandschaft sollen einheitliche Servicelevels für Forschungsdaten und $\mathrm{OA}$ sowie juristische und technische Standards für den Umgang mit Forschungsdaten geschaffen werden. Die Sichtbarkeit der nationalen Forschungsleistung soll mittels offener Ressourcen für die Forschungscommunity, und mit der Schaffung von Grundlagen, um semantikbezogene Anwendungen entwickeln zu können (Linked Data), erhöht werden. Dabei bildet sowohl ein Forschungsdatenmanagement als Voraussetzung für Antragstellerlnnen als auch die universitätsinterne Vernetzung die Basis für zukünftiges Einwerben nationaler und internationaler Projekte.

Der Cluster A des Projekts „Monitoring und Austausch zum Aufbau von Dokumentservern in den lokalen Einrichtungen" wurde näher vorgestellt und der aktuelle Stand der Arbeiten präsentiert.

Zielsetzungen sind das Monitoring sowie der rege Austausch vor, während und nach der Implementierung von Repositorien, in denen sowohl Digital Born Publications, Digitalisate als auch Bilder abgelegt werden können.

Dabei ist die Erstellung eines modularen Anforderungskataloges zum Aufbau lokaler Infrastrukturen sowie einer modularen Arbeitsbeschreibung für einen zukünftig tätigen Repository Manager bereits zu 95\% 
abgeschlossen. Konzepte für das Monitoring sind zu $50 \%$, Konzepte für Workshops zum Austausch zu 20\% entwickelt. Mit der Erstellung einer Marktübersicht wurde begonnen (10\%). Erste Veröffentlichungen wurden für 14 Tage nach der Informationsveranstaltung in Aussicht gestellt. Alle Dokumente werden unter CC-BY veröffentlicht. Alle Projektpartner sollen am Ende ein eigenes Repository einrichten können. Ergebnisse aus dem gesamten Projekt sollen frei zur Verfügung gestellt werden.

Nationale Standards für Repositorien in Österreich wurden in der anschließenden Diskussion als unrealistisch angesehen, da einzelnen Forschungsstätten in diesem Zusammenhang keine Vorschreibungen gemacht werden können.

\section{Abschlussdiskussion}

In einer abschließenden Diskussionsrunde wurde danach mit den anwesenden Personen die Zukunft von OANA diskutiert. Falk Reckling leitete die Runde mit der Frage nach dem Weiterbestehen und den möglichen Formen des Fortbestands ein. Vier Szenarien erschienen ihm dabei möglich:

1. Aufarbeitung der AGs und dann Beendigung des Netzwerks,

2. Thematik wie bisher weiterführen und Vertiefung der AG-Themen bzw. Gründung neuer AGs,

3. Ausweitung der Thematik auf u.a. Open Data, Open Science,

4. Hybridform zwischen zweiter und dritter Option.

Bei einer Weiterführung sei besonders das Nebeneinander von sich ähnelnden Initiativen zu vermeiden und Überlegungen zur einer längerfristigen Finanzierung notwendig.

Neben den organisatorisch-administrativen Anforderungen wurden auch Überlegungen zur Erweiterung des Themenportfolios bei OANA angestellt. Ein möglicher Bereich wäre „Open Science“ (Open Data, Open Methodology, Open Peer Review oder Open Educational Ressources), den Peter Kraker (KNOW Center Graz) und Michaela Vignoli ( $\underline{\text { IIT }})$ vorstellten.

In der anschließenden Diskussion wurde deutlich, dass von allen Anwesenden eine Weiterführung des Open Access Network Austria sehr stark befürwortet wird. Die OANA wurde als wertvolles und professionelles österreichisches Netzwerk gelobt, das sich auch in Zukunft inhaltlich vordergründig des Themas Open Access annehmen sollte. Der Bereich Open Data könnte langsam in das Aufgabenfeld der OANA einfließen, würde aber zum jetzigen Zeitpunkt - vor allem in Bezug auf technische Aspekte - den Themenbereich des Netzwerks sprengen. Beim Thema Open Sci- 
ence waren sich viele Anwesende einig, dass dies derzeit andernorts besser aufgehoben sei. Konsens herrschte darüber, dass Überschneidungen mit anderen österreichischen Initiativen, wie beispielsweise e-infrastractures Austria, vermieden werden sollten. In diesem Zusammenhang wurde auch die Erstellung eines möglichen Fahrplans zum Thema Open Access in Österreich angemerkt. Dadurch könnten Ziele besser definiert und die Finanzierung sowie der Personaleinsatz besser strukturiert werden. Sehr positiv hervorgehoben wurde das Format von OANA, das aus einer Zusammensetzung von Networking Sessions und Hands-on so oder in ähnlicher Form weitergeführt werden sollte. Handlungsbedarf wurde in der Kommunikation mit den VizerektorInnen gesehen und eine Neubeauftragung durch das Ministerium über die Leistungsvereinbarung an den Universitäten erwünscht.

Am Ende des Veranstaltungstags fasste Falk Reckling die zwei wichtigsten Aufgaben für die kommenden Monate noch einmal zusammen. Es müssten einerseits die in der Veranstaltung gesammelten Informationen aufgearbeitet und publiziert werden und andererseits in einem OANAKernteam Treffen ein Vorschlag ausgearbeitet werden, in welcher Form OANA weitergeführt werden sollte.

Katrin Buschmann, MSc Fonds zur Förderung der wissenschaftlichen Forschung (FWF) E-Mail: katrin.buschmann@fwf.ac.at

Katharina Rieck, MA Fonds zur Förderung der wissenschaftlichen Forschung (FWF) E-Mail: katharina.rieck@fwf.ac.at

In der Aufarbeitung der Ergebnisse der Veranstaltung hat nun das Kernteam für die nächsten 12-15 Monate zwei Arbeitsgruppen zur Weiterführung und drei neue Arbeitsgruppen empfohlen:

- Einbindung von Wissenschafterlnnen in Open Access Weiterführung der bestehenden Arbeitsgruppe;

Koordinatoren: Michael Nentwich (ÖAW) und Michael Strassnig (WWTF)

- Publikationskosten

Weiterführung der bestehenden Arbeitsgruppe mit Fokus auf

a) Erhebung, Administration und Umwidmung von Publikationskosten zu Open Access sowie 
b) Koordination der Verhandlungen mit Verlagen.

Koordinator: Falk Reckling (FWF)

- Urheberrecht

neue Arbeitsgruppe, in der Vorschläge für ein wissenschaftsfreundlicheres Urheberrecht ausgearbeitet werden sollen.

Koordinator: Wolfgang Nedobity (UNIKO)

- Nationale Strategie

neue Arbeitsgruppe, die an einem Entwurf füreine nationale Open Access Strategie arbeitet.

Koordinator: Peter Seitz (BMWFW)

- Open Access/Open Science und Scholarly Communication neue Arbeitsgruppe, die diskutiert, wie Open Access und Open Science die wissenschaftliche Kommunikation langfristig verändert und bestehende Defizite beheben können.

Koordinator: Peter Kraker (Open Knowledge Foundation, KNOW Center Graz)

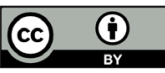

Dieses Werk ist lizenziert unter einer Creative-Commons-Lizenz Namensnennung 3.0 Österreich. 